\title{
PERSONAL COMPUTER-BASED DIGITAL PETROPHYSICS
}

\author{
Ricardo Leiderman ${ }^{1}$, Andre M.B. Pereira², Francisco M.J. Benavides ${ }^{1}$, Carla S. Silveira ${ }^{3}$, \\ Rodrigo M.R. Almeida² and Rodrigo A. Bagueira ${ }^{3}$
}

\begin{abstract}
In the present work, we describe our experience with digital petrophysics, enhancing our choices for performing the related tasks. The focus is on the use of ordinary personal computers. To our best knowledge, some of the information and hints we give cannot be found in the literature and we hope they may be useful to researchers that intend to work on the development of this new emerging technology. We have used micro-scale X-ray computed tomography to image the rock samples and, in that sense, we address here the issue of the corresponding image acquisition and reconstruction parameters adjustment. In addition, we discuss the imaging resolution selection and illustrate the issue of the representative volume choice with the aid of two examples. The examples corroborate the notion that it is much more challenging to define a representative volume for carbonate samples than for sandstone samples. We also discuss the image segmentation and describe in details the Finite Element computational implementation we developed to perform the numerical simulations for estimating the effective Young modulus from segmented microstructural images. We indicate the respective computational costs and show that our implementation is able to handle comfortably images of $300 \times 300 \times 300$ voxels. We use a commercially available Finite Volume software to estimate the effective absolute directional permeability.
\end{abstract}

Keywords: rock physics, micro-scale X-ray computed tomography, multi-scale homogenization, effective elastic moduli, representative volume.

RESUMO. No presente trabalho descrevemos nossa experiência com Petrofísica Digital, dando ênfase às nossas escolhas para a realização das tarefas relacionadas. 0 foco é no uso de computadores pessoais e, salvo melhor juízo, algumas das informações e dados que apresentamos não podem ser achados na literatura. Nós adquirimos as imagens digitais de amostras de rochas com 0 auxílio de microtomografia computadorizada por raio- $\mathrm{X}$ e, nesse sentido, discutimos aqui 0 ajuste dos parâmetros de aquisição e reconstrução de imagens. Além disso, nós discutimos a questão da seleção do volume representativo e sua relação com o tamanho e resolução da imagem digital, mostrando dois exemplos ilustrativos. Os exemplos corroboram a noção de que é muito mais difícil definir um volume representativo tratável para carbonatos do que para arenitos. Nós também discutimos a segmentação de imagens no contexto da Petrofísica Digital e descrevemos em detalhes o código de Elementos Finitos por nós desenvolvido para estimar o módulo de Young efetivo de amostras de rochas a partir de suas imagens microtomográficas, indicando 0 respectivo custo computacional. Nós mostramos que nossas escolhas levaram a uma implementação computacional capaz de lidar confortavelmente com imagens de até $300 \times 300 \times 300$ voxels. Por fim, descrevemos o uso do pacote comercial de Volumes Finitos para estimar a permeabilidade absoluta efetiva das amostras de rocha.

Palavras-chave: física de rochas, microtomografia computadorizada por raio-X, homogeneização multiescala, módulo de Young efetivo, volume representativo.

\footnotetext{
${ }^{1}$ Universidade Federal Fluminense - UFF, Departamento de Ciências da Computação, Av. Gal. Milton Tavares de Souza, s/n, São Domingos, 24210-346 Niterói, RJ, Brazil. E-mails: leider@ic.uff.br; fbenavides@id.uff.br

2 Universidade Federal Fluminense - UFF, Escola de Engenharia, Rua Passo da Pátria, 156, São Domingos, 24210-253 Niterói, RJ, Brazil.

E-mails: andremaues@id.uff.br; rraposo@id.uff.br

3 Universidade Federal Fluminense - UFF, Instituto de Química, Outeiro de São João Batista, s/n, 24020-141 Niterói, RJ, Brazil.

E-mails: carla@geoq.uff.br; rbagueira@vm.uff.br
} 


\section{INTRODUCTION}

The use of micro-scale X-ray computed tomography $(\mu \mathrm{xCT})$ to image rock samples has experienced an exponential growth in the last two decades. The early attempts to use this technology for rock imaging, which goes beyond the 2D microscopy in the definition of the $3 \mathrm{D}$ rock microstructure, started at the end of the XX century and were mainly restricted to the use of synchrotron radiation (Flannery et al., 1987). The development of X-ray laboratory tube sources at the beginning of the XXI century, levered by a high demand from the petroleum industry, allowed $\mu \mathrm{XCT}$ devices to be installed and operated in small facilities, making it more popular for sedimentary rock microstructure description and classification (see, e.g., Cnudde et al., 2006; Cnudde et al., 2011; Lander \& Bonnell, 2015; Mostaghimi et al., 2013; Mees et al., 2003 and Van Geet et al., 2003), and for reservoir rock analysis (see, e.g., Cnudde et al., 2011 and Wood et al., 2011).

The feasibility of using $\mu \mathrm{xCT}$ for imaging rock samples has opened new possibilities in the area of Petrophysics and nowadays numerical simulations can be performed from highresolution microstructural 3D rock images in the sense of simulating physical processes of interest to estimate effective rock properties as well as to investigate in detail local petrophysical mechanisms. To the collection of mathematical and computational procedures associated to these tasks we conventionally call Digital Petrophysics (or Digital Rock Physics). This new emerging technology has been developed as a common effort between the Academia and the oil companies and has gradually become more important in the area. It has been mainly applied to the analysis of sandstones, although the discovery of pre-salt reservoir rocks in the Brazilian continental margin sedimentary basin has increased the demand for specific studies targeting carbonate analog rocks (see e.g. Machado et al., 2015 and Oliveira et al., 2012).

Generally speaking, digital petrophysics always involves the association of three main distinct steps (Andra et al., 2013b):

i) digital image acquisition;

ii) digital image processing; and

iii) numerical simulation performed from processed digital image.

Each one of these steps presents its own specific challenges, intrinsic to its corresponding concentration area, that are still open tasks. Accordingly, we understand the development of this new technology as an interdisciplinary effort whose success depends on the association of researchers coming from different areas such as Engineering, Physics, Geology and Computer Science.
Our research group has the capability of performing each one of these three mentioned steps. In that sense, the main goal of the present article is to describe our experience with digital petrophysics, enhancing our choices for performing the related tasks. The focus here is on the use of ordinary personal computers (PCs), since most of the research groups spread around the globe do not have access to fancy clusters or super computers. To our best knowledge, some of the information and hints we give cannot be found in the literature and we hope they may be useful to researchers that intend to work on the development of digital petrophysics. Our methodology, from sample preparation to effective petrophysical parameters estimation, passing through specimen mounting, data acquisition, reconstruction, segmentation, model mesh generation and computational simulation, is depicted schematically in Figure 1.

In the next section, we describe our experience with $\mu \mathrm{xCT}$ and digital image acquisition and reconstruction. Next, we discuss the issue of imaging resolution selection and image segmentation in Sections 3 and 4, respectively. In Section 5, we discuss the computational implementations used to perform the numerical simulations for estimating the effective Young modulus and the effective absolute directional permeability of rock samples. Then, we conclude in Section 6.

\section{MICRO-CT DATA ACQUISITION AND IMAGE RECONSTRUCTION}

Radiography is an imaging technique that uses $\mathrm{X}$-ray to reveal material inner structure and composition. In this technique, that is essentially 2D, the sample to be imaged is positioned between an X-ray source and a detector, as schematically represented in Figure 2(a). Roughly speaking, the attenuation on the energy that is transmitted through the sample and registered by the detector is proportional to the sample's density. In general, denser parts will absorb more energy. Therefore, the energy registered by the detector reflects the sample's density spatial distribution. Tomography, that is essentially 3D, is based on the radiographic repetitive sectioning of the sample, where radiograms are sequentially acquired while the sample (or the source-detector system) is incrementally rotated, as schematically indicated in Figures 2(a) and 2(b). From the collection of the acquired radiograms, crosssectional images of the sample are computed using tomographic reconstruction algorithms, producing a stack of 2D raw gray-scale horizontal image slices, as indicated in Figure 2(c). The 3D digital object can then be visualized from the 2D gray-scale image stack, as indicated in Figure 2(d). In $\mu \mathrm{xCT}$, the dimensions of the reconstructed voxels (i.e. a volumetric pixel element) are in general in the micrometer range. 


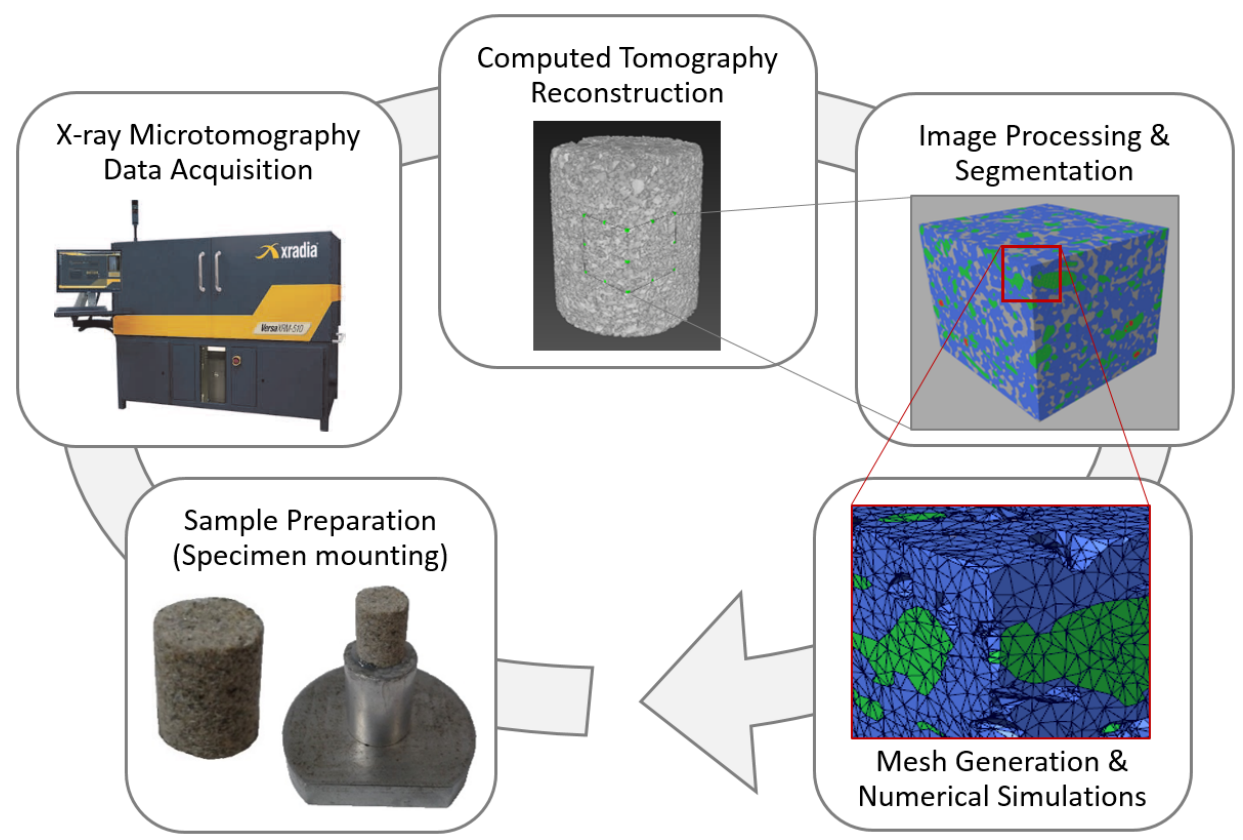

Figure 1 - Our methodology, from sample preparation to numerical results, passing through specimen mounting, data acquisition, reconstruction, segmentation, model mesh generation and computational simulations.

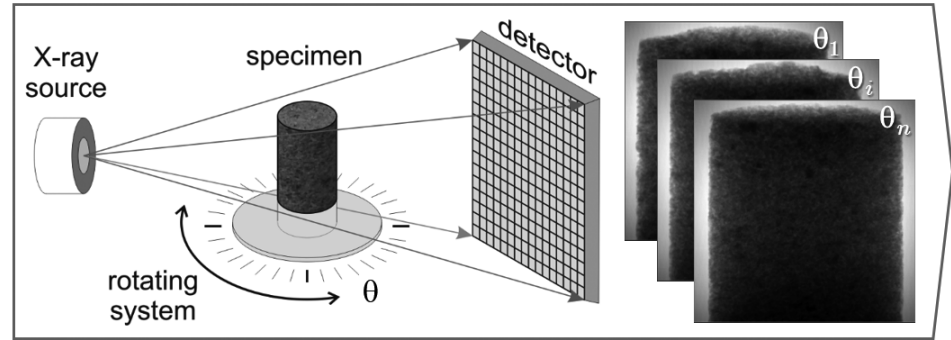

(a) (b)

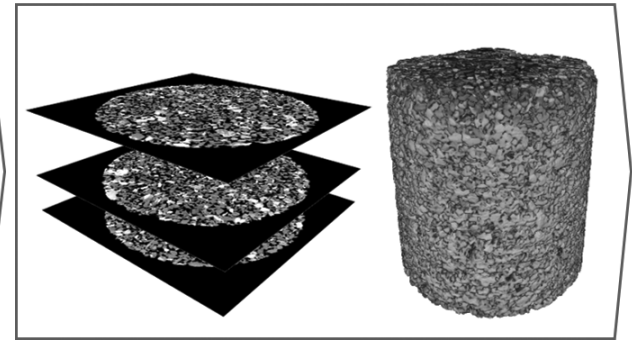

(c)

Figure 2 - Illustrative scheme of CT acquisition workflow: (a) setup of $\mu \mathrm{xCT}$ scanner for image acquisition, (b) set of single radiographic projections, (c) stack of 2D raw gray-scale horizontal image slices, and (d) volume reconstructed.

We have used the $\mu$ xCT system Xradia 510 Versa, made by ZEISS, to image the rock samples. In this system, there are different options for magnifying lens (0.4X, 4X,20X and 40X) and it is possible to adjust the positioning of both source and detector in relation to the sample. In addition, during the acquisition, the sample rotates around its axis while the source-detector set remains fixed. Usually, we work with cylindrical samples and glue them to a cylindrical sample holder (steel support) using resin. The samples are mounted so that the X-ray beam is perpendicular to the cylinder axis, as shown in Figure 2(a). Sample drift during the acquisition will affect the quality of the reconstructed image. In that sense, even that it may be (in part) subsequently corrected, we always run a fast scan (that usually takes half an hour) prior of running the imaging scan, in order to warm up the system, stabilizing the enclosure temperature and humidity, reducing thus the possibility for drifting. The acquisition scanning parameters to be adjusted by the user are binning, magnification (objective lens), source-sample-detector positioning, source voltage/wattage, source filter type, exposure time, and number of projections. We discuss each one along with its respective adjustment in the following, grouping the parameters according to the image feature that they most influence. At this point, it is important to say that the adjustment of each parameter is connected, directly or indirectly, to the adjustment of all other parameters, and often we must go forward and backward to achieve a satisfactory overall adjustment. 


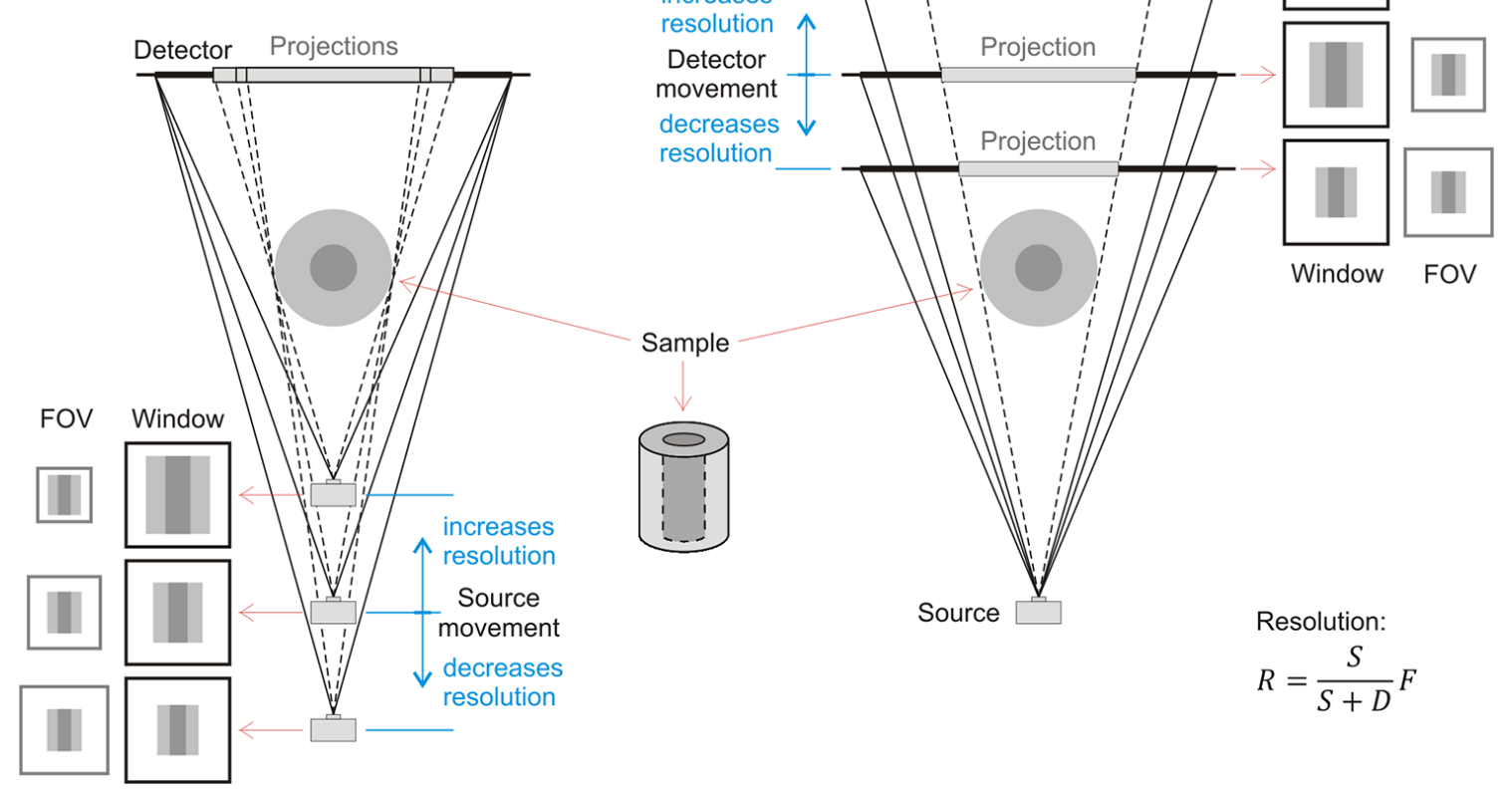

Figure 3 - The relation of the source-sample and detector-sample distances with the image resolution.

\section{Image resolution}

The source-sample and the detector-sample distances are two of the parameters that determine the image resolution. The sample's portion illuminated by the source and projected on the detector is usually called Field of View (FOV). Moving either the detector closer to the sample or the source away from the sample increases the FOV, thus reducing the image resolution. Conversely, moving either the detector away from the sample or the source closer to the sample increases the image resolution. This idea is illustrated schematically in Figure 3. In our system, the maximum sourcesample distance is $195 \mathrm{~mm}$ while the maximum source-detector distance is $335 \mathrm{~mm}$. In addition to the source-sample-detector positioning, magnifying lens may be placed in front of the detector to increase the resolution. As mentioned before, four options for magnifying lens are available $-0.4 \mathrm{X}, 4 \mathrm{X}, 20 \mathrm{X}$ and 40X. The fourth parameter that straightforwardly contributes to the image resolution is the BIN (or binning) rate. Binning is the procedure of combining a cluster of pixels into a single pixel. It is a computational procedure rather than an "optical" effect. When binning rate is set to 2 , for example, the visual light camera averages 4 pixels (a two by two matrix) into a single pixel. Therefore, the image number of pixels (and the resolution) is reduced by a factor of 4 . In our system, each projection can be captured with BIN 1, BIN 2 , BIN 4 or BIN 8. The default is the $2048 \times 2048$ 2-Byte detector array (BIN 1), although for rock samples the $1024 \times 1024$ 2-Byte detector array (BIN 2) is the most used. A single projection with BIN 1 is nearly 8.4 MB while with BIN 2 it is reduced to $2.1 \mathrm{MB}$. The image resolution will be then determined by the conjunction of the four parameters discussed here and can be calculated with the aid of the formula shown at the lower right of Figure 3. In the formula, $R$ is the pixel dimension, $S$ is the source-sample distance, $D$ is the source-detector distance, and $F$ is a factor that takes into account the detector specifications, the selected lens, and the selected BIN rate. In our system, for example, $F=68.93 \mu \mathrm{m}$ for $\mathrm{BIN}=2$ and Lens $=0.4 \mathrm{X}$

\section{Image brightness and contrast}

The image brightness and contrast basically depend on two factors: i) the X-ray energy that is transmitted through the sample and reaches the detector, which is straightforwardly linked to the selected source voltage/wattage and to the sample's density and thickness; and ii) the exposure time. In practice, these parameters are adjusted iteratively. We begin by choosing an initial value for the source voltage $-80 \mathrm{kV}$ (at maximum of $7 \mathrm{~kW}$ ) for lowdensity materials and/or thinner samples or $140 \mathrm{kV}$ (at maximum $10 \mathrm{~kW}$ ) for high-density materials and/or thicker samples. The source current is then automatically selected by the system. Next, we check the percentage of the source energy that is transmitted 
through the sample. The goal here is to have the transmission rate in the range of $20 \%-35 \%$. To that end, we may use a filter if necessary and/or correct the source voltage/wattage. Last, we adjust the exposure time. This parameter is adjusted in order to have at minimum a value of 5000 for the exposure intensity. For reservoir rocks, the exposure time usually varies from 1 to 5 sec for each frame.

The parameters discussed above are related to the radiogram (a single projection) features. The tomogram is reconstructed from a collection of radiograms. The number of radiograms used in the reconstruction straightforwardly affects the 3D final image quality. On the other hand, it is directly linked to the total scanning time, since the last can be roughly estimated as the exposure time $\times$ number of projections. According to our experience, good quality images can be obtained with 1600 projections (the sample is rotated by angular increments of $0.225^{\circ}$ ) that results in a total amount of collected data of $3.36 \mathrm{~GB}$ (BIN 2) or $13.44 \mathrm{~GB}$ (BIN 1). However, data storage can reach 12.6 GB with BIN 2 for high quality images (6000 projections). It is yet worth mentioning that there is a technique called "secondary reference" that may further improve the image quality, if desired. In addition, there is a technique known as "dual energy" that is used to characterize the chemical composition of the sample by scanning it at two different energy levels.

Following the acquisition, the $\mu \mathrm{xCT}$ datasets are computationally processed in order to produce a 3-D digital object, as depicted schematically in Figures 2(c) and 2(d). This step is called image reconstruction, and two user-defined reconstruction parameters are critical for the reconstructed image quality: the Center Shift Correction (CSC) and the Beam Hardening Correction $(\mathrm{BHC})$. In practice, each one is adjusted based on a rule of thumb. The CSC value is chosen from the visual comparison of several reconstructions of the same horizontal slice, each one performed with a different correction value. The selected CSC value will be then the one that gives the sharpest image. The BHC value, in turn, is chosen taking into account the gray-scale value associated to the background mineral phase. In that sense, again, we compare several reconstructions of the same horizontal slice, each one performed with a different correction value. More specifically, we analyze the pixel gray-scale along a line that crosses each one of the reconstructions. Considering that the background mineral does not change along this line, we select the BHC value that gives the overall flatter plot. The issue of the BHC adjustment is illustrated with the aid of Figure 4. In the figure, the three gray-scale plots shown at the upper half are associated to the "plot line" that crosses the reconstructions shown at the lower half. Each recon- struction was performed with a different BHC value. We selected the $\mathrm{BHC}$ value corresponding to the middle plot, and performed the subsequent 3D object reconstruction accordingly. Notice that the plot on the left is curved up, while the plot on the right is curved down. As a final comment, we emphasize that for the CSC and BHC adjustments, only the horizontal slice used in the comparison is reconstructed rather than the full 3D object.

We end this section presenting tomograms in Figures 5 and 6 along with the corresponding values for the acquisition and reconstruction parameters. The tomograms shown in Figure 5 correspond to a sandstone, while the tomograms shown in Figure 6 correspond to a carbonate. The values for the corresponding acquisition and reconstruction parameters are shown in Tables 1 and 2 , respectively.

\section{IMAGING RESOLUTION SELECTION}

The image resolution plays a central role in the success of the subsequent analysis presented in Section 5. Generally speaking, the resolution should be selected based on two antagonistic criteria:

i) it should be high enough to resolve well the microstructure;

ii) it should be low enough such that it allows to pick a representative volume whose corresponding image size is treatable in the numerical simulation procedures.

At this point, we should say in advance that in the present work we consider as treatable images of up to $300 \times 300 \times 300$ voxels. We illustrate the issue of imaging resolution selection in the two following examples.

In Figures 5(a) and 5(b), we see two $\mu \times C T$ slices of the same sandstone micro-plug. In Figure 5(a), the plug was imaged with a resolution of $5 \mu \mathrm{m}$, while in Figure $5(\mathrm{~b})$ it was imaged with a resolution of $1 \mu \mathrm{m}$. Both slices have 1024 pixels of diameter. The red solid line in Figure 5(a) delimits a square region composed by $300 \times 300$ pixels and Figure 5 (c) is a magnified view of this region. By visual inspection of Figure 5(a), we see that the slice's microstructure is roughly homogeneous and that any other $300 \times 300$-pixel square region chosen within the slice would have approximately the same microstructure. In that sense, we may say that for this resolution a $300 \times 300 \times 300$ voxel cube can be taken as a representative volume (we have verified that the microstructure do not differ significantly from slice to slice in the 3D image represented in Figure 5(d)). In addition, we see that we can distinguish well the grain contours in Figure 5(c), indicating that the resolution of $5 \mu \mathrm{m}$ is enough to resolve the plug's microstructure. 


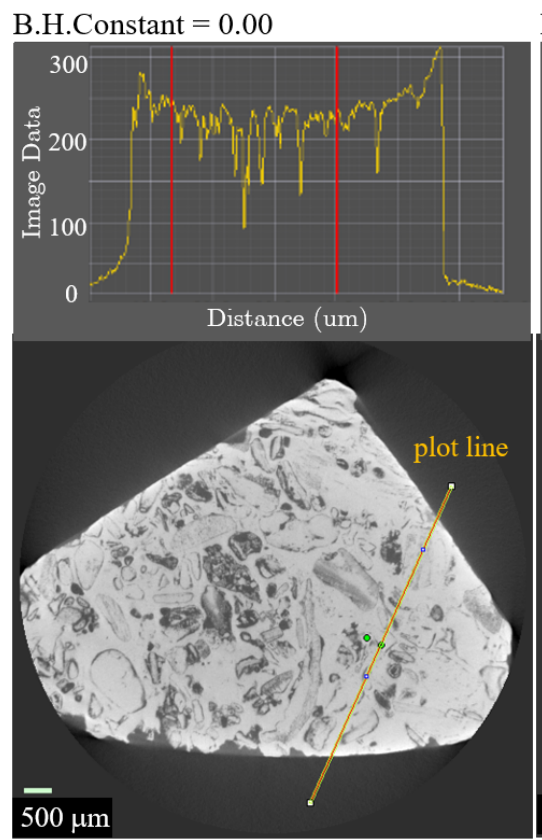

B.H.Constant $=0.12$

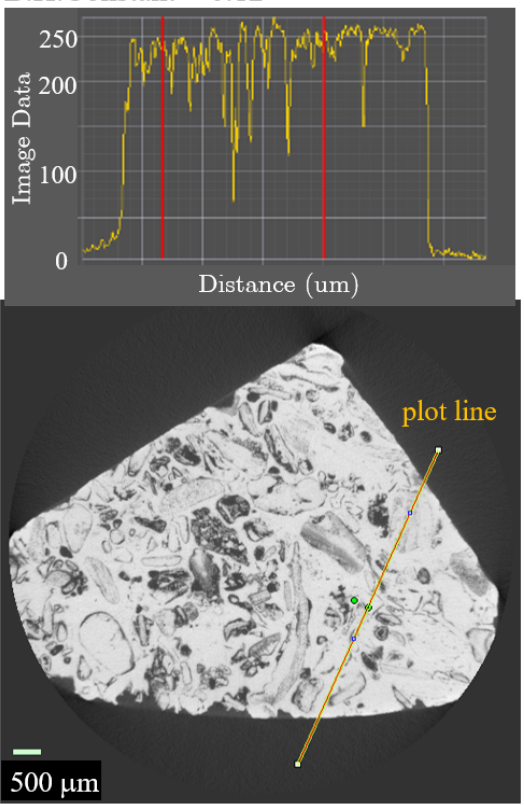

B.H.Constant $=0.50$

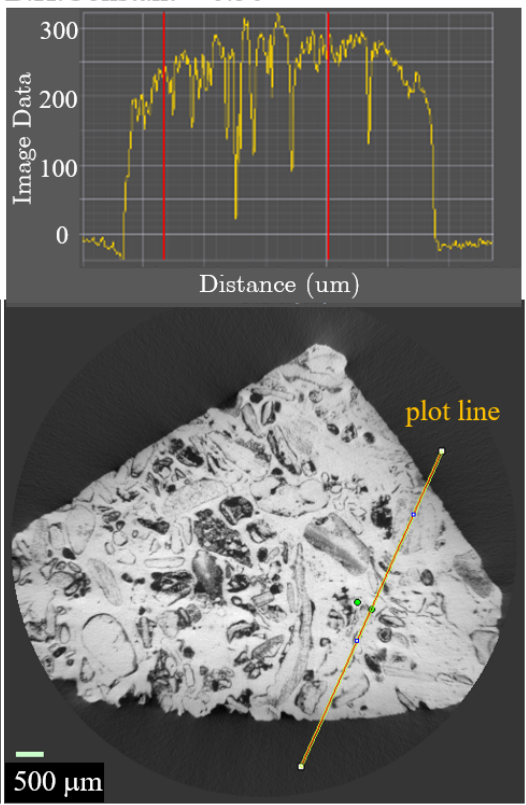

Figure 4 - The Beam Hardening Correction (BHC) value selection. The three reconstructions shown at the lower half were performed with a different BHC value. The corresponding crossing line gray-scale plots are shown at the upper half. The BHC value corresponding to the middle plot was the selected one.

We conclude then that this resolution is suitable for the present case, since it meets both criteria mentioned above. The yellow dashed line delimiting a square region in Figure $5(\mathrm{~b})$ is also composed by $300 \times 300$ pixels. In corroboration with what was seen for the resolution of $5 \mu \mathrm{m}$, we see that the resolution of $1 \mu \mathrm{m}$ is enough to resolve the microstructure. In fact, we see much better the grain contour details in this figure than we see in Figure 5(c), although we believe that these details would not impact significantly the numerical simulations. On the other hand, we see now that there are just a few (parts of) grains within the square region, and that other $300 \times 300$-pixel regions chosen within the slice would potentially have a very different microstructure. This indicates that for the resolution of $1 \mu \mathrm{m}$ a $300 \times 300 \times 300$ voxel cube can no longer be considered as a representative volume, thus indicating that this resolution is not suitable for our purposes. We also have imaged the same micro-plug with a resolution of $10 \mu \mathrm{m}$ and have verified that with this resolution we were no longer able to resolve well the microstructure.

In Figures 6(a) and 6(b), we see two $\mu \mathrm{xCT}$ slices of the same carbonate micro-plug. In Figure 6(a), the plug was imaged with a resolution of $10 \mu \mathrm{m}$, while in Figure $6(\mathrm{~b})$ it was imaged with a resolution of $5 \mu \mathrm{m}$. By visual inspection, we see in the figures that even the $10 \mu \mathrm{m}$ resolution was enough to resolve reasonably well the microstructure. Again, the red solid line in Figure 6 (a) and the yellow dashed line in Figure $6(\mathrm{~b})$ delimit a square region composed by $300 \times 300$ pixels. Differently from before, however, we see in Figure 6(a) that the slice's microstructure is not homogeneous and that other $300 \times 300$-pixel regions chosen within the slice would possibly have a very different microstructure. In that sense, we may say that for this resolution a $300 \times 300 \times 300$ voxel cube is not a representative volume (of course, the same occurs for the $5 \mu \mathrm{m}$ resolution). We conclude then that there is not a resolution that meets both criteria mentioned in the previous paragraph. This example corroborates the consensual notion that carbonates tend to be much more heterogeneous then sandstones and that, therefore, it is much more challenging to define a representative volume for carbonate samples then for sandstone samples. To estimate effective properties for this sample, one would have to perform the corresponding numerical experiments for several different "subimages" of the original 3D image and try to combine the results using some specific homogenization or statistical rule. This is far beyond the scope of the present work.

\section{IMAGE SEGMENTATION}

In the context of digital petrophysics, the main goal of image segmentation is to identify (and label) all the distinct phases in the rock sample digital image. However, for purposes of porosity and effective permeability calculation, we only need to define the pore 


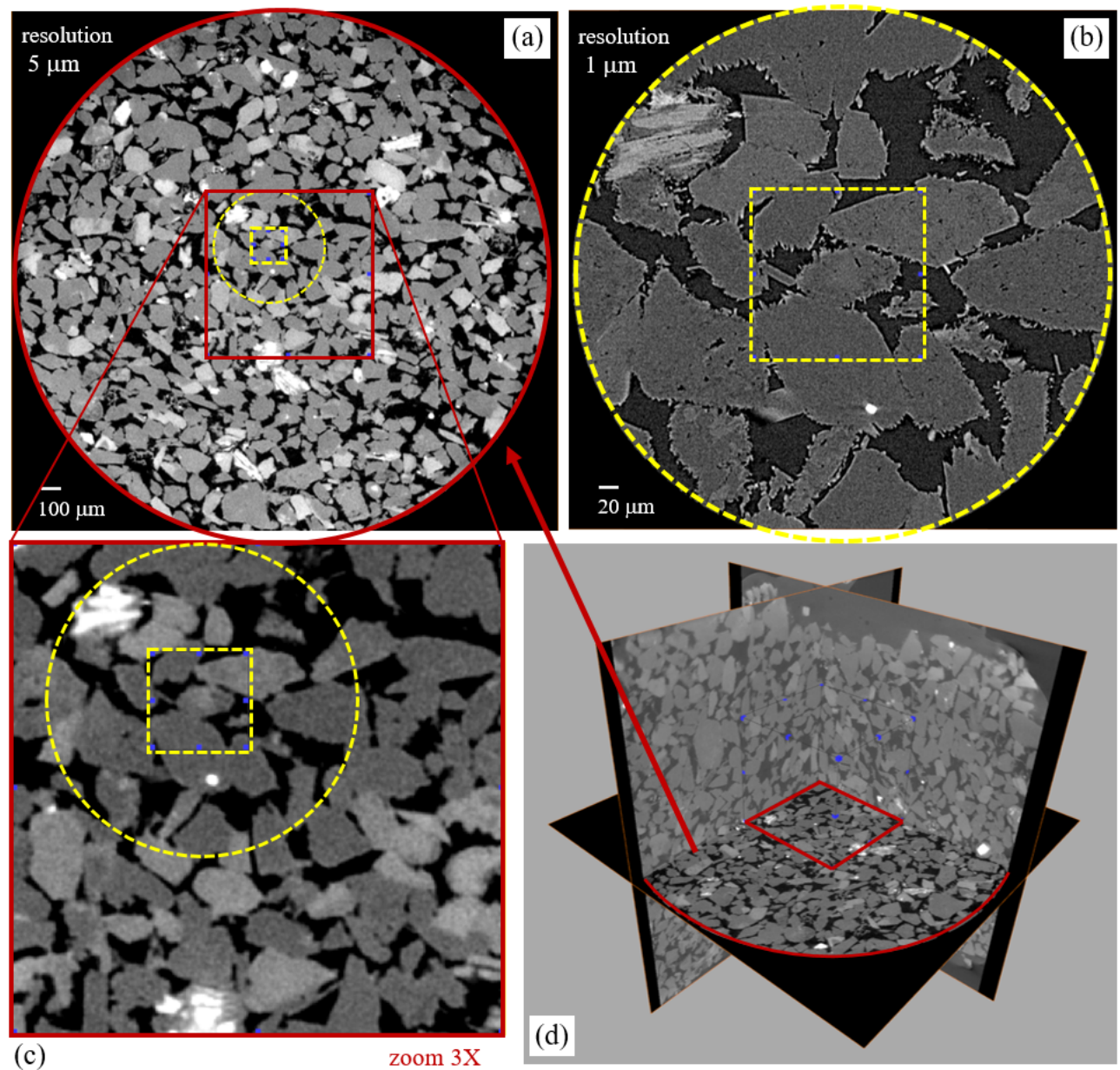

Figure $\mathbf{5}$ - Slices of two tomograms obtained from the same sandstone sample. (a) $5 \mu \mathrm{m}$ of resolution. (b) $1 \mu \mathrm{m}$ of resolution. (c) Magnified view of the $300 \times 300$-pixel square region delimited by the red solid line in (a). (d) Orthogonal cross-sections of the $5 \mu \mathrm{m}$-resolution tomogram. The values for the corresponding acquisition and reconstruction parameters are shown in Tables 1 and 2, respectively.

space. To do that, a simple thresholding procedure may be applied in order to generate a binary image, where one color is associated to the solid skeleton and another color is associated to the pore space. Computing the porosity reduces then to count the total number of voxels corresponding to the pore space and divide that number by the image total number of voxels. On the other hand, for the effective elastic moduli calculation we need to identify all distinct mineral phases. The simplest way for doing that is to apply a multiple thresholding procedure, where, based on the histogram analysis, each defined gray-scale range corresponds to a mineral phase. Usually, it works well for high quality images and it has been our routine procedure for segmentation. More sophisticated image segmentation techniques are those based on the region-growing concept, where small seed regions are chosen and grown by some specific rules (see, e.g., Andra et al., 2013a; Sakellariou et al., 2007, and references therein). These techniques take much longer to be applied, demanding a computational cost much larger than the one demanded by the multiple thresholding technique, and usually cannot automatically segment the tomogram. In addition, they (slightly) modify the original reconstructed image as they synthetically (re)define the rock microstructure. In that sense, they must be applied with caution in order to not introduce artifacts and to preserve all the rock sample significant original microstructural features.

Regardless of the segmentation method, a denoising filter should be applied prior to the segmentation process itself for improving the reconstructed image quality. There are several different filters for this purpose and we usually use the non-local 

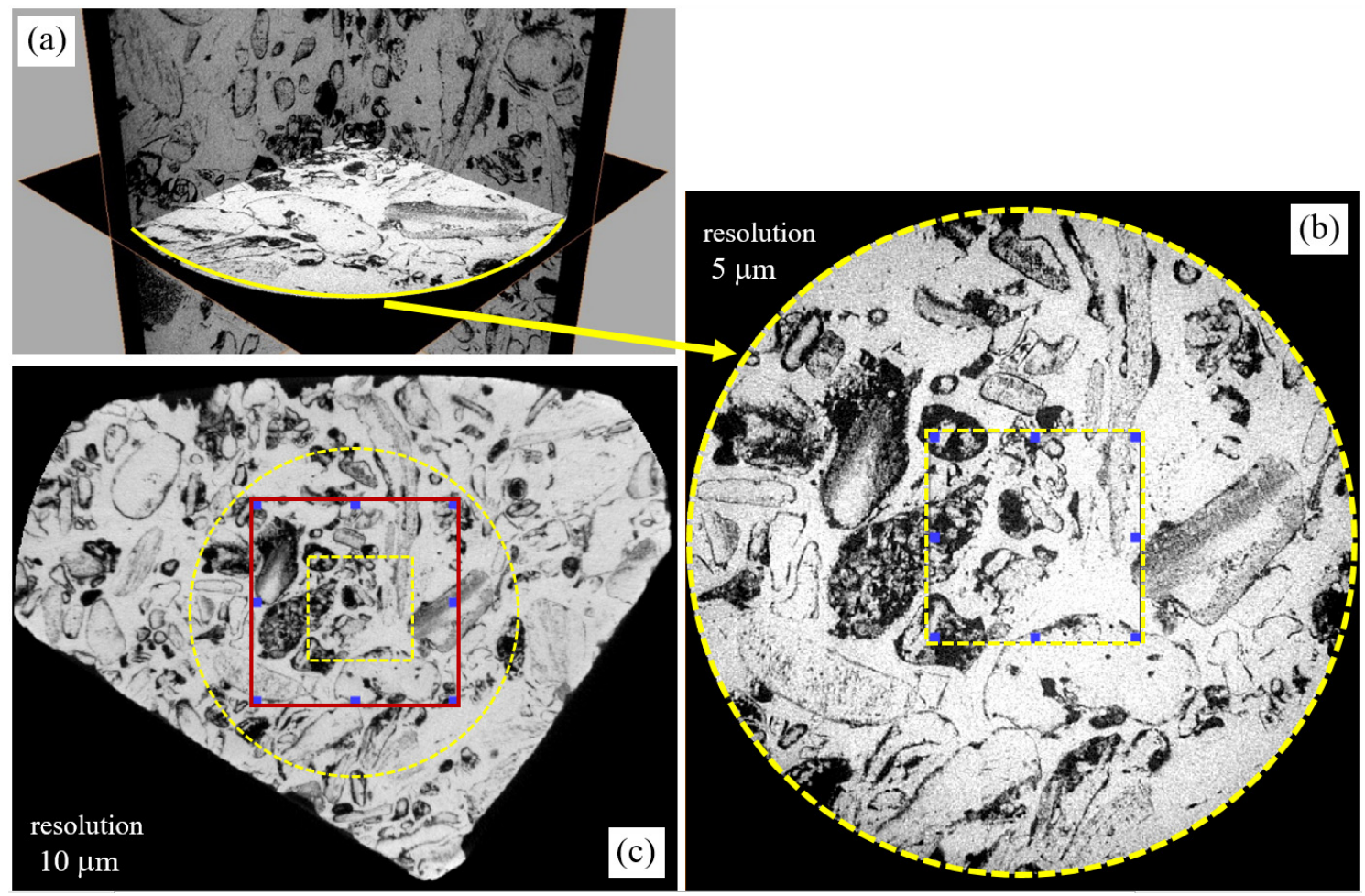

Figure 6 - Slices of two tomograms obtained from the same carbonate sample. (a) Orthogonal cross-sections of the $5 \mu$ m-resolution tomogram. (b) $5 \mu \mathrm{m}$ of resolution. (c) $10 \mu \mathrm{m}$ of resolution. The values for the corresponding acquisition and reconstruction parameters are shown in Tables 1 and 2, respectively.

Table 1 - Scanning parameters used for the tomograms shown in Figures 5 and 6.

\begin{tabular}{|l|c|c|c|l|c|c|c|c|}
\hline $\begin{array}{l}\text { Sample } \\
\text { material } \\
\text { (lens) }\end{array}$ & $\begin{array}{c}\text { Source- } \\
\text { sample } \\
\text { Dist. } \\
(\mathrm{mm})\end{array}$ & $\begin{array}{c}\text { Detector- } \\
\text { sample } \\
\text { Dist. } \\
(\mathrm{mm})\end{array}$ & $\begin{array}{c}\text { Resolut. } \\
\text { Voxel } \\
\text { size } \\
(\mu \mathrm{m})\end{array}$ & Filter & $\begin{array}{c}\text { Source } \\
\text { Voltage } \\
\text { Power } \\
\text { Current }\end{array}$ & $\begin{array}{c}\text { Expos. } \\
\text { Time }\end{array}$ & $\begin{array}{c}\text { Number } \\
\text { project. }\end{array}$ & $\begin{array}{c}\text { Scan. } \\
\text { time } \\
(\mathrm{h})\end{array}$ \\
\hline $\begin{array}{l}\text { Sandstone } \\
\text { IB (4X) }\end{array}$ & 55 & 19 & 5,015 & LE\#2 & $\begin{array}{c}70 \mathrm{kV} \\
6 \mathrm{~W} \\
86 \mathrm{uA}\end{array}$ & $3.0 \mathrm{~s}$ & 6001 & $\sim 5.0$ \\
\hline $\begin{array}{l}\text { Sandstone } \\
\text { IB (4X) }\end{array}$ & 15 & 85 & 1,013 & LE\#2 & $\begin{array}{c}70 \mathrm{kV} \\
6 \mathrm{~W} \\
86 \mathrm{uA}\end{array}$ & $4.7 \mathrm{~s}$ & 5001 & $\sim 6.5$ \\
\hline $\begin{array}{l}\text { Carbonate } \\
\text { EY (0.4X) }\end{array}$ & 30 & 177 & 10,000 & LE\#4 & $\begin{array}{c}70 \mathrm{kV} \\
6 \mathrm{~W} \\
86 \mathrm{uA}\end{array}$ & $3.5 \mathrm{~s}$ & 1601 & $\sim 1.5$ \\
\hline $\begin{array}{l}\text { Carbonate } \\
\text { EY (4X) }\end{array}$ & 71 & 25 & 5,000 & LE\#4 & $\begin{array}{c}70 \mathrm{kV} \\
7 \mathrm{~W}\end{array}$ & $10.0 \mathrm{~s}$ & 1601 & $\sim 4.5$ \\
\hline
\end{tabular}


Table 2 - Reconstruction parameters used for the tomograms shown in Figures 5 and 6.

\begin{tabular}{|l|c|c|c|}
\hline $\begin{array}{c}\text { Sample material } \\
\text { (lens) }\end{array}$ & $\begin{array}{c}\text { Resolut. Voxel } \\
\text { size }(\mu \mathrm{m})\end{array}$ & $\begin{array}{c}\text { Center } \\
\text { Shift }\end{array}$ & $\begin{array}{c}\text { Beam } \\
\text { Hardening }\end{array}$ \\
\hline Sandstone IB (4X) & 5,015 & 6.0 & 0.80 \\
Sandstone IB (4X) & 1,013 & 14.38 & 0.05 \\
Carbonate EY (0.4X) & 10,000 & 4.8 & 0.12 \\
Carbonate EY (4X) & 5,000 & 15.0 & 0.00 \\
\hline
\end{tabular}

means image smoothing filter that removes the high-frequency noise. Another option is the three-dimensional anisotropic diffusion filter, as mentioned in Andra et al. (2013a), which, in addition to removing the high-frequency noise, aims also to preserve the grain contours and sharp features in the image. Both for image filtering and for image (multiple) thresholding we have used a commercially available image processing package that is capable of handling $2048 \times 2048 \times 2048$ voxel images in a very short amount of time, using an ordinary PC.

\section{NUMERICAL SIMULATIONS}

\section{Effective elastic parameters}

We estimate the effective elastic parameters with the aid of the Finite Element Method. The Finite Element Method is widely used in the context of computational solid mechanics and is a numerical method mainly applied to solve (partial) differential equations. It is based on the weak (variational) formulation of the problem and turns the original problem into a corresponding algebraic system from the domain discretization. Here, we developed and implemented a 3D Finite Element code to solve the Elastostatic Equation using the classical formulation corresponding to the Galerkin approximation and tri-linear shape functions. The code was implemented in $\mathrm{C}_{++}$and we used OpenMP and ITBB (Intel Threading Building Blocks) extensions for parallelism. We give more details bellow.

\section{Mesh}

The first step in the method application is the physical domain discretization. This step is also known as "meshing" and generally the domain is meshed using polygonal elements. Here, we mesh the domain using the concept that each voxel (pixel) in the digital image will correspond to a tri (bi)-linear element in the mesh, resulting in a regular mesh (all the elements are cubes of the same size). The physical dimension of each element is linked to the image resolution. Therefore, differently from what happens in other application areas, the computational costs to mesh the domain here are extremely low. On the other hand, the linear systems resulting from this procedure are usually very large. Finite Element implementations based on this meshing procedure are sometimes referred as Voxel-Based Finite Element Method (VFEM).

Based on what is described above, we developed and implemented a $\mathrm{C}++$ code that automatically builds a regular mesh from the segmented image, assigning the elastic parameters to each element according to the color of the corresponding voxel. Notice that this gives us the flexibility to easily represent the grain geometries, as well as to assign appropriate elastic parameters to different mineral constituents or grain contours. Further, we have the option of not meshing the pore space, resulting in a mesh corresponding only to the solid skeleton. Therefore, we are able to simulate tests on dry as well as on saturated rock. For the elastic parameters estimation, we perform the simulations on dry rock.

\section{Finite Element Implementation}

The second step in the method application is the computation of the entries on the right and left hand side (RHS \& LHS) of the linear system. As we mentioned before, we expect to deal with large linear systems. For instance, if the inputted 3D digital image is composed by $300 \times 300 \times 300$ voxels, we expect to have a system with about 90 millions of degrees of freedom. In that sense, the stiffness matrix (the LHS of the system) would have $O\left(10^{9}\right)$ non-null elements. It would require at least $50 \mathrm{~GB}$ for its memory storage using double precision, which is more than we have available in an ordinary PC. For that reason, we have opted for a special implementation usually called "Element by Element" (EBE) implementation (or EBE implicit method) that takes full advantage of the fact that all elements in the mesh are of the same size and that never really assemble a vector with all the non-null elements of the LHS and RHS of the system, as described in Hughes (2000) and in Smith \& Griffiths (2004). In this special implementation, we work solely in the "element domain". In that sense, we expect 
to be able to deal with large meshes, although our implementation is not the fastest possible. It is a trade-off between speed and memory space. In addition, as it is common for the element by element implementation, we solve the resulting linear system (that we never really assemble) with the aid of the Gradient-Conjugate Method. Here, we use the implementation described in Fong \& Saunders (2012) with Jacobi's preconditioning.

\section{Parallelization}

In any finite element implementation there are linear algebra procedures that can be parallelized. These procedures encompass common operators for vectors and matrices: product, addition, scalar product, etc. Most common workstations have several processors in shared memory architecture and ordinary vector operation such as addition can be easily distributed across them, making each one work on a particular vector section. In addition, in the EBE implementation mentioned above the computations for each element can be performed in parallel. However, the tasks should not be distributed arbitrarily between the processors since two processors working with two different elements that share the same node may try to update the same vector entry simultaneously, causing memory contention or even dirty read/write. For that reason, in the present implementation we divide the elements into different "groups" (or "colors") such that the elements of the same group do not share nodes, as it is schematically represented in Figure 7(c). Within each group, the computations are then performed in parallel, although the groups are processed sequentially. For our $3 \mathrm{D}$ regular mesh where each voxel corresponds to an (cube) element, we need to divide the elements into 8 different groups (4 different groups for a 2D implementation). It is yet worth mentioning that, in principle, this procedure might be implemented for GPUs as well.

\section{Estimating Effective Elastic Parameters}

In the present work, we assume that the sample is isotropic. Accordingly, only two elastic parameters are needed to define its constitutive behavior. In what follows, we will consider the effective Young modulus and the effective Poisson ratio of the sample. Basically, there are two different numerical approaches for estimating the effective elastic parameters. The first one is simply reproducing the unconfined compression test (UCT) as it is performed in physical lab. This test consists in applying an uniaxial compression load to the specimen and measuring the resulting overall strain. The boundary conditions for the corresponding numerical simulation are the following: The specimen's bottom cannot move in the $y$ (vertical) direction, but can freely lat- erally expand; The specimen's laterals are considered as free surfaces where, accordingly, the components of the corresponding stress vectors are all null; We prescribe a uniform vertical displacement $u_{y}$ at the specimen's top, while it can freely laterally expand. The numerical simulation with the corresponding boundary conditions are shown schematically in Figure 7(a). The effective Young modulus can then be calculated as the ratio between the overall applied vertical strain and the corresponding (computed) compression load. The effective Poisson ratio may be inferred from the ratio between the axial and transversal overall strain. See Chevalier et al. (2007), Harrison et al. (2008), Niebur et al. (2000), Rietbergen et al. (1995), and Ulrich et al. (1998) for examples of application with this approach.

The second approach comes from the multi-scale homogenization and consists in using the so-called Periodic Boundary Conditions (PBC). The basic assumption behind the use of $\mathrm{PBC}$ is that the numerical model is a representative volume of an unbounded (statistically homogeneous) medium (Hashin, 1983). In the context of the Finite Element Method, the PBC are applied assigning the same equation number to corresponding nodes at opposite sides. See Nguyena et al. (2012) for implementation details and Arns et al. (2002), Garboczi \& Day (1995), Garboczi \& Berryman (2001), Makarynska et al. (2008), and Roberts \& Garboczi (2002) for examples of application with this approach. The numerical simulation with the corresponding boundary conditions are shown schematically in Figure 7(b).

Both methods mentioned above have been commonly used to estimate effective elastic parameters. Roughly speaking, the unconfined compression test simulation is indicated for cases where the input image physical dimensions are much larger than the microstructure characteristic length, and it demands a computational cost shorter than the one demanded by the use of PBC. Estimates provided by both approaches get closer as the physical dimension of the image gets larger in comparison to the microstructure characteristic length. Our implementation takes about 15 minutes to simulate the unconfined compression test for a $100 \times 100 \times 100$ voxel input image, takes about 2 hours for a $200 \times 200 \times 200$ voxel image, takes about 10 hours for a $300 \times 300 \times 300$ voxel image, takes about 1 day for a $400 \times 400 \times 400$ voxel image, and we estimate that it would take about 4 days to simulate the unconfined compression test for a $500 \times 500 \times 500$ voxel input image.

\section{Effective directional absolute permeability}

Analogously to what was discussed in the previous section, here there are also two different numerical approaches to estimate the 


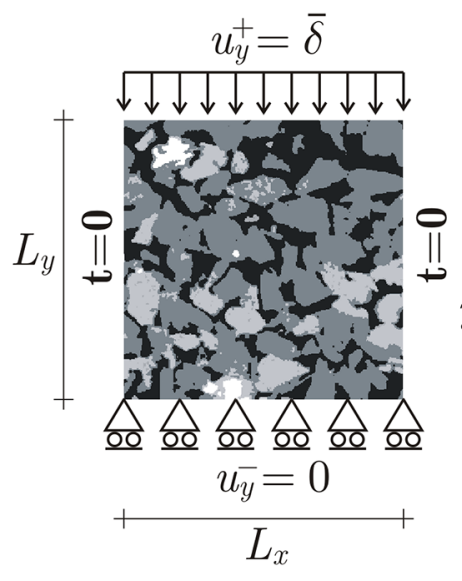

(a)

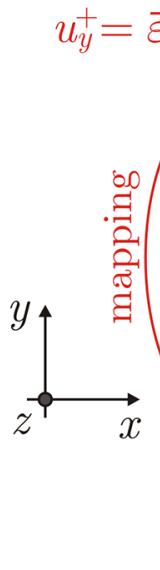

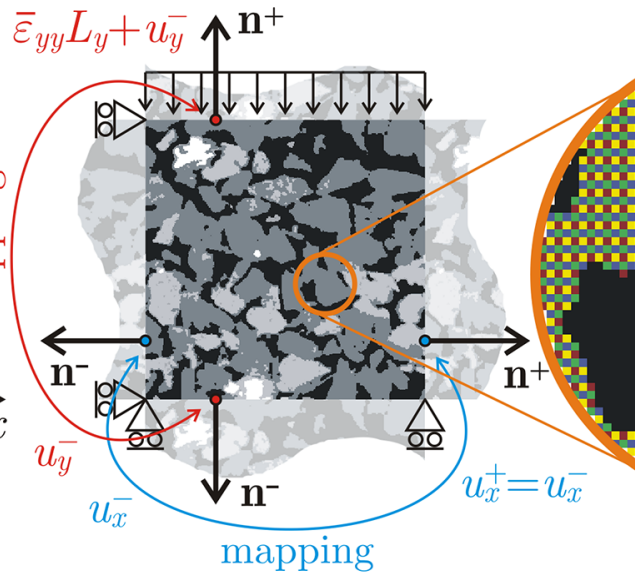

(b)

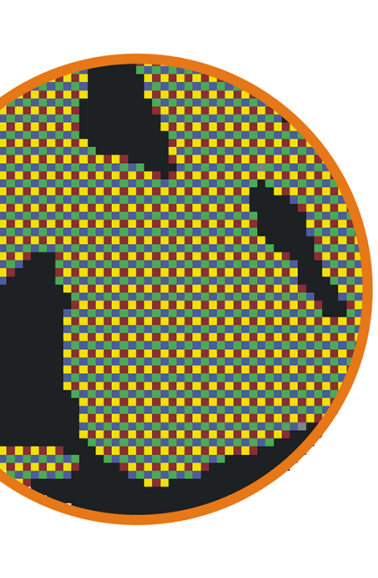

(c)

Figure 7 - (a) Boundary conditions used in the unconfined compression test numerical simulation. (b) Periodic Boundary Conditions (PBC). (c) Voxel-based finite element mesh with greedy coloring.

permeability. The first one is simply reproducing the permeability test as it is performed in physical lab. Concisely, this test consists in applying a directional pressure gradient across the specimen and measuring the flow rate at the steady state. Accordingly, the imposed boundary conditions in the corresponding numerical simulation are the following: fluid cannot flow across two pairs of opposite faces, indicating that the sample is hermetically sealed on these four faces. The pressure is set on the two opposite remaining faces, such as the pressure is constant at each face but larger at one face than at the other. Therefore, a pressure gradient is stated across the sample. When the steady state is reached, the ratio between the flow rate and the prescribed pressure jump is used in the Darcy's law to determine the effective permeability. The simulation may be repeated for the other two remaining directions. As before, the second approach consists in imposing spatially periodic boundary conditions, analogously to what is schematically depicted in Figure 7(b). The basic assumption behind the use of PBC is that the numerical model is a representative volume of an unbounded (statistically homogeneous) medium. Again, the simulation of the permeability test as it is performed in a physical lab is indicated for cases where the input image physical dimensions are much larger than the microstructure characteristic length, and it demands a computational cost shorter than the one demanded by the use of PBC. The estimates provided by both procedures get closer as the physical dimension of the image gets larger (in comparison to the microstructure characteristic length).

We perform the permeability estimations using both numerical approaches discussed above with the aid of a commer- cially available Finite Volume software. It is a module of the image processing package mentioned at the end of Section 3 and, in that sense, it builds automatically the Finite Volume mesh from the segmented image. For the first approach mentioned above, it takes around 20 minutes for performing the estimation with a $100 \times 100 \times 100$ voxel image, takes around 3 hours for a $200 \times 200 \times 200$ voxel image, and takes around 12 hours for performing the estimation with a $300 \times 300 \times 300$ voxel image. Our computing power was not enough to run simulations with $400 \times 400 \times 400$ voxel images. For the second mentioned approach $(\mathrm{PBC})$, the Finite Volume software takes around twice Ionger to perform the simulations.

\section{SUMIMARY AND CONCLUSIONS}

In the present work, we described our experience with digital petrophysics, enhancing our choices for performing the related tasks using ordinary personal computers. We discussed $\mu \mathrm{xCT}$ digital image acquisition and reconstruction, addressing the issue of the related parameters adjustment, and discussed the imaging resolution selection and image segmentation. We illustrated the issue of the representative volume pick with the aid of two examples that corroborate the notion that it is much more challenging to define a representative volume for carbonate samples than for sandstone samples. In addition, we described in details the computational implementations that we use to perform the numerical simulations for estimating the effective Young modulus and the effective absolute directional permeability from segmented microstructural images of rock samples. 
For the effective Young modulus, we have implemented a C++ Finite Element code that handles comfortably images of $300 \times 300 \times 300$ voxels. For the absolute permeability, we use a commercial Finite Volume software and are not able to performe the corresponding numerical simulations from images larger than $300 \times 300 \times 300$ voxels. We hope the information and hints we give here may be useful to researchers that intend to work in the development of this new emerging technology.

\section{ACKNOWLEDGMENTS}

This work was carried out in association with the ongoing NMR Project, sponsored by Shell, registered as "Aplicação de técnicas avançadas de Ressonância Magnética Nuclear (RMN) assistidas por ferramentas computacionais na avaliação petrofísica de rochas carbonáticas" (Agência Nacional do Petróleo, Gás Natural e Biocombustíveis - ANP 18999-3) under the ANP R\&D levy as "Compromisso de Investimentos com Pesquisa e Desenvolvimento". The authors also recognize the support from Coordenação de Aperfeiçoamento de Pessoal de Nível Superior (Capes), Conselho Nacional de Desenvolvimento Científico e Tecnológico (CNPq) and Fundação Carlos Chagas Filho de Amparo à Pesquisa do Estado do Rio de Janeiro (FAPERJ). F.B. recognizes the support from Universidad Nacional de Costa Rica (UNA).

\section{REFERENCES}

ANDRA H, COMBARET N, DVORKIN J, GLATT E, HAN J, KABEL M, KEEHM Y, KRZIKALLA F, LEE M, MADONNA C, MARSH M, MUKERJI T, SAENGER EH, SAIN R, SAXENA N, RICKER S, WIEGMANN A \& ZHAN X. 2013a. Digital rock physics benchmarks-Partl: Imaging and segmentation. Computers \& Geosciences, 50: 25-32.

ANDRA H, COMBARET N, DVORKIN J, GLATT E, HAN J, KABEL M, KEEHM Y, KRZIKALLA F, LEE M, MADONNA C, MARSH M, MUKERJI T, SAENGER EH, SAIN R, SAXENA N, RICKER S, WIEGMANN A \& ZHAN X. 2013b. Digital rock physics benchmarks-Part II: Computing effective properties. Computers \& Geosciences, 50: 33-43.

ARNS CH, KNACKSTEDT MA, PINCZEWSKI WV \& GARBOCZIEJ. 2002. Computation of linear elastic properties from microtomographic images: Methodology and agreement between theory and experiment. Geophysics, 67(5): 1396-1405.

CHEVALIERY, PAHR D, ALLMER H, CHARLEBOIS M \& ZYSSETP. 2007. Validation of a voxel-based FE method for prediction of the uniaxial apparent modulus of human trabecular bone using macroscopic mechanical tests and nanoindentation. Journal of Biomechanics, 40: 3333-3340.

CNUDDE V, MASSCHAELE B, DIERICK M, VLASSENBROECK J, VAN HOOREBEKE $L$ \& JACOBS P. 2006. Recent progress in X-ray CT as a geosciences tool. Applied Geochemistry, 21: 826-832.
CNUDDE V, BOONE M, DEWANCKELEJ, DIERICK M, VAN HOOREBEKE $L$ \& JACOBS P. 2011. 3D characterization of sandstone by means of $X$-ray computed tomography. Geosphere, 7(1): 54-61.

FLANNERY BP, DECKMAN HW, ROBERGE WG \& D'AMICO KL. 1987. Three-dimensional X-ray microtomography. Science, 237: 1439-1444.

FONG DC \& SAUNDERS M. 2012. CG versus MINRES: An empirical comparison. SQU Journal for Science, 17(1): 44-62.

GARBOCZIEJ \& DAY AR. 1995. An algorithm for computing the effective linear elastic properties of heterogeneous materials: three-dimensional results for composites with equal phase poisson ratios. Journal of the Mechanics and Physics of Solids, 43(9): 1349-1362.

GARBOCZIEJ \& BERRYMAN JG. 2001. Elastic moduli of a material containing composite inclusions: effective medium theory and finite element computations. Mechanics of Materials, 33: 455-470.

HARRISON NM, MCDONNELL PF, O'MAHONEY DC, KENNEDY OD, O'BRIEN FJ \& MCHUGH PE. 2008. Heterogeneous linear elastic trabecular bone modelling using micro-CT attenuation data and experimentally measured heterogeneous tissue properties. Journal of Biomechanics, 41 : 2589-2596.

HASHIN Z. 1983. Analysis of composite materials. Journal of Applied Mechanics, 50: 481-505.

HUGHES T. 2000. The Finite Element Method - Linear Static and Dynamic Finite Element Analysis (Mineola, NY: Dover), 483-487.

LANDER RH \& BONNELL LM. 2015. Predicting sediment and sedimentary rock properties. United States Patent Application Publication US 2015/0355158A1, $71 \mathrm{pp}$.

MACHADO AS, DAL BÓ PFF, LIMA I, BORGHI L \& LOPES RT. 2015. $X$-ray microtomography characterization of carbonate microbialites from a hypersaline coastal lagoon in the Rio de Janeiro State-Brazil. Nuclear Instruments and Methods in Physics Research A, 784: 574-580.

MAKARYNSKA D, GUREVICH B, CIZ R, ARNS CH \& KNACKSTEDT MA. 2008. Finite element modelling of the effective elastic properties of partially saturated rocks. Computers \& Geosciences, 34: 647-657.

MEES F, SWENNEN R, VAN GEET M \& JACOBS P. 2003. Applications of $\mathrm{X}$-ray computed tomography in the geosciences. Geological Society, London, Special Publications, 215: 1-6.

MOSTAGHIMI P, BLUNT MJ \& BIJELJIC B. 2013. Computations of Absolute Permeability on Micro-CT Images. Mathematical Geosciences, 45: 103-125.

NGUYENAV-D, BÉCHETAE, GEUZAINEB C \& NOELS L. 2012. Imposing periodic boundary condition on arbitrary meshes by polynomial interpoIation. Computational Materials Science, 55: 390-406.

NIEBUR GL, FELDSTEIN MJ, YUEN JC, CHEN TJ \& KEAVENY TM. 2000. High-resolution finite element models with tissue strength asymmetry 
accurately predict failure of trabecular bone. Journal of Biomechanics, 33: $1575-1583$.

OLIVEIRA MFS, LIMA I, BORGHI L \& LOPES RT. 2012. X-ray microtomography application in pore space reservoir rock. Applied Radiation and Isotopes, 70: 1376-1378.

RIETBERGENB, WEINANS H, HUISKES R \& ODGAARDT A. 1995. A new method to determine trabecular bone elastic properties and loading using micromechanical finite-element models. Journal of Biomechanics, 28(1): 69-81.

ROBERTS AP \& GARBOCZI EJ. 2002. Computation of the linear elastic properties of random porous materials with a wide variety of microstructure. Proceedings of the Royal Society of London A, 458: 1033-1054.

SAKELLARIOU A, ARNS CH, SHEPPARD AP, SOK RM, AVERDUNK A, LIMAYE A, JONES AC, SENDEN TJ \& KNACKSTEDT MA. 2007. Devel- oping a virtual materials laboratory. Materials Today, 10(12): 44-51.

SMITH IM \& GRIFFITHS DV. 2004. Programming the Finite Element Method. West Sussex, England: John Wiley \& Sons Ltd., 64-67.

ULRICH D, RIETBERGENB, WEINANS H \& RUEGSEGGERP. 1998. Finite element analysis of trabecular bone structure: a comparison of imagebased meshing techniques. Journal of Biomechanics, 31: 1187-1192.

VAN GEET M, LAGROU D \& SWENNEN R. 2003. Porosity measurements of sedimentary rocks by means of microfocus $X$-ray computed tomography $(\mu \mathrm{CT})$. In: MEES F, SWENNEN R, VAN GEET M \& JACOBS P (Eds.). Applications of $X$-ray computed tomography in the geosciences. Geological Society, London, Special publications, 215: 51-60.

WOOD R, CURTIS A \& KAYSER A. 2011. Classification method for sedimentary rocks. United States Patent US7869565B2, 12 pp. 